\title{
Entero-vesical fistula revealed by recurrent urinary tract infection
}

\begin{abstract}
Colo-vesical fistula is a common complication of diverticular disease of sigmoid colon. The most common presenting symptom is pneumaturia, followed by dusuria, fecaluria, abdominal pain and, rarely, hematuria. We herein report an incidental diagnosis of colovesical fistula during a cystography performed for etiological assessment of a recurrent urinary tract infection in a 76-years-old patient emphasizing clinical aspects, diagnostic methods and management.
\end{abstract}

Keywords: fistula; diverticulitis; cystography; urinary infection

\author{
Volume 6 Issue 4 - 2018 \\ Youness Jabbour, ${ }^{1,2}$ Youssef Lamzaf, ${ }^{1,2}$ Tarik \\ Karmouni, ${ }^{1,2}$ Khalid El Khader, ${ }^{1,2}$ Abdellatif \\ Koutani, ${ }^{1,2}$ Ahmed Iben Attya Andaloussi ${ }^{1,2}$ \\ 'Urology B department, Ibn Sina teaching hospital, Rabat, \\ Morocco \\ ${ }^{2}$ Faculty of medicine and pharmacy, Mohammed V University, \\ Rabat, Morocco
}

Correspondence: Youness Jabbour, Resident at Urology B department, Ibn Sina teaching hospital, Hay El Manzah N 800 C.Y.M, I0I50, Rabat, Morocco, Tel $00212(0) 660278360$, Email uro.jabbour@gmail.com

Received: August 09, 2018 | Published: August 17, 2018

\section{Introduction}

Entero-vesical fistulas are defined by the existence of an abnormal pathway that communicates the bladder with the intestine which may be colovesical, rectovesical, ileovesical or appendicovesical. ${ }^{1,2}$ Initially described in 1685, Colo-vesical fistula is the most common form of vesico-intestinal fistula and is most frequently located between the sigmoid colon and the dome of the bladder. ${ }^{2,3}$ We report a case of a patient incidentally diagnosed with colo-vesical fistula while performing an etiological diagnosis of a recurrent urinary tract infection.

\section{Case report}

Our patient is 76-years-old with no particular past medical history complaining about recurrent symptomatic urinary tract infections. He reported pollakuria, dysuria and intermittent diarrhea without any accompanying fever or hematuria.

Clinical examination finds a patient in good general health, afebrile with vital signs were within normal limits (Blood pressure: 124/68mmHg, Heart rate: 68 beats/minute, Respiratory rate 15 breath/ minute, $\mathrm{SaO}_{2} 99 \%$ on ambient air). Rectal examination revealed a tender prostate of small size. An abdominopelvic ultrasound found a bladder wall thickening with a small prostate making 22g. Postvoid residual urine volume was not significant. A cytobacteriological examination of the urine revealed a urinary infection with E. coli.

An antibiotherapy was started and retrograde and urinary uretrocystography was performed. Retrograde cystography showed diverticular bladder with opacification of the sigmoid colon up to the half of the transverse colon (Figure 1) (Figure 2).

Recto-sigmoidoscopy failed to find the orifice of the fistula. Abdominopelvic computed tomography showed a circumferential thickening of the bladder wall with a posterior parietal collection of $11 * 16 \mathrm{~mm}$ in contact with the sigmoid colon. Uncomplicated colonic diverticula were also revealed (Figure $3-5$ ).

The patient underwent an open laparotomy. Surgical exploration revealed an intimate contact between sigmoid colon and the bladder dome with thickening in both structures. Sigmoidectomy was performed taking away vesical peri-fistular area with immediate restoration of continuity by colorectal anastomosis. Post-operatory was uneventful. Histologic examination revealed an inflammatory process without signs of malignancy. The patient was put on medical treatment for his obstructive urinary symptomatology with good evolution.

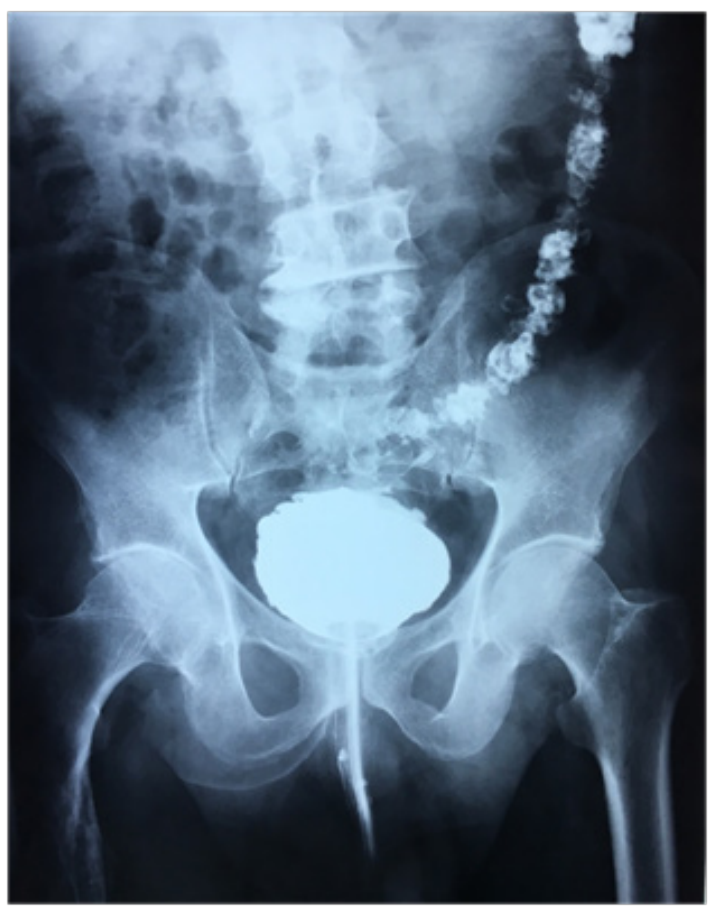

Figure I Frontal view of retrograde urethrocystography showing opacification of the sigmoid and descending colon. 


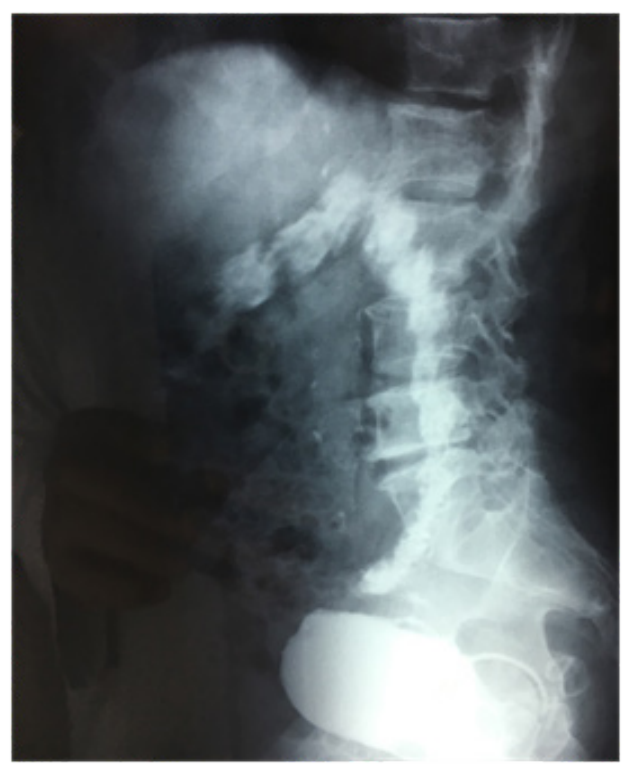

Figure 2 Profile view of retrograde urethrocystography showing opacification of colon until half of his transverse portion.

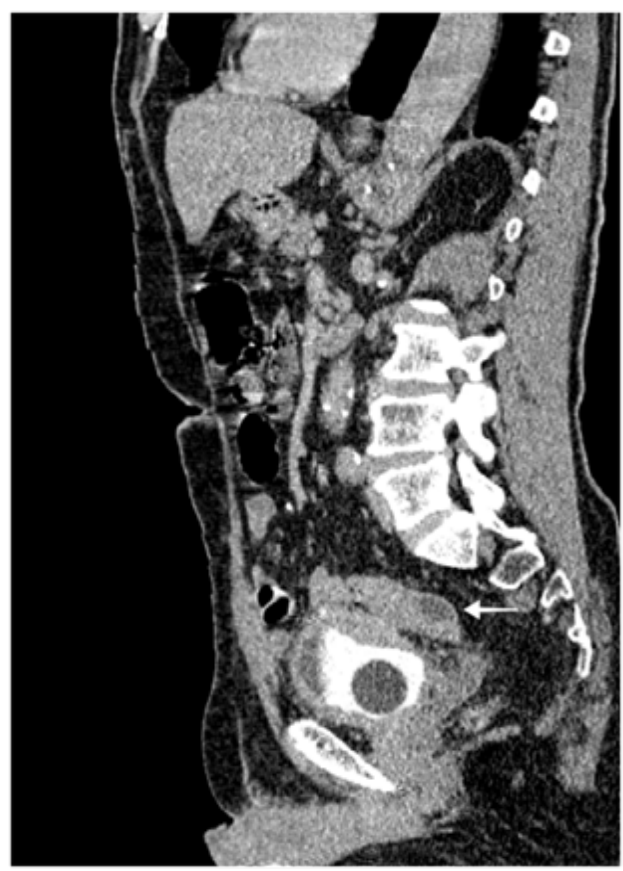

Figure 3 Sagittal computed tomography scan showing retro vesical collection with close contact with sigmoid colon and bladder thickening its posterior wall. (Arrow)

\section{Discussion}

Entero-vesical fistula is most often the consequence of an inflammatory and infectious process of digestive origin that wills secondarily fistulize in an initially healthy bladder. Diverticulitis represents the commonest etiology of entero-vesical fistula accounting for approximately $65-79 \%$ of cases, which are almost exclusively colo-vesical. ${ }^{2}$ Neoplasia accounting for $15 \%$ to $46 \%$ of cases represents the second most common cause of entero-vesical fistula followed by Crohn's disease in $7 \%$ to $15 \%$ of cases. ${ }^{3}$ More rarely, these entero-vesical fistulas can be of radic, post-traumatic or iatrogenic origin. ${ }^{4}$

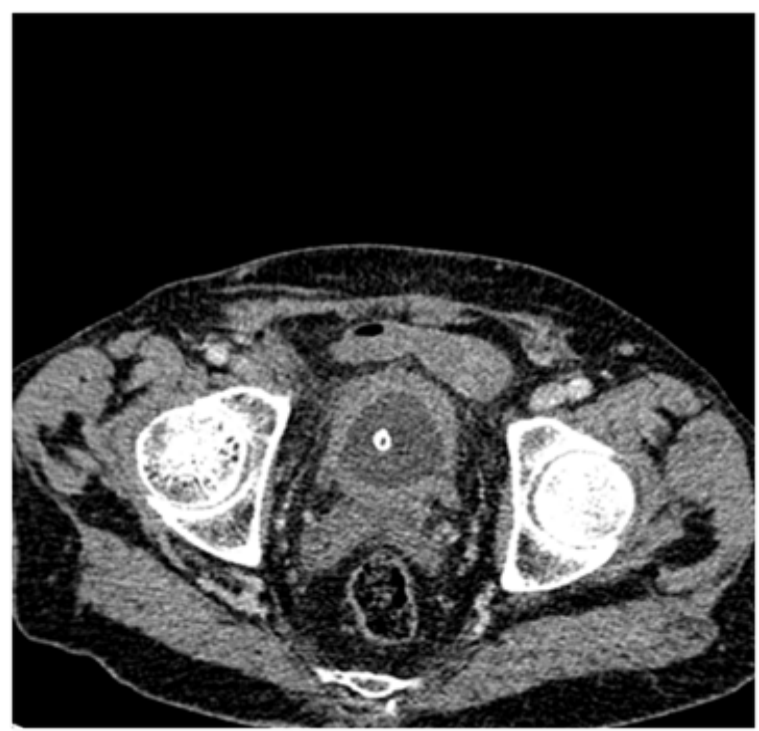

Figure 4 Transversal computed tomography scan showing circumferential bladder wall thickening because of urinary obstruction.

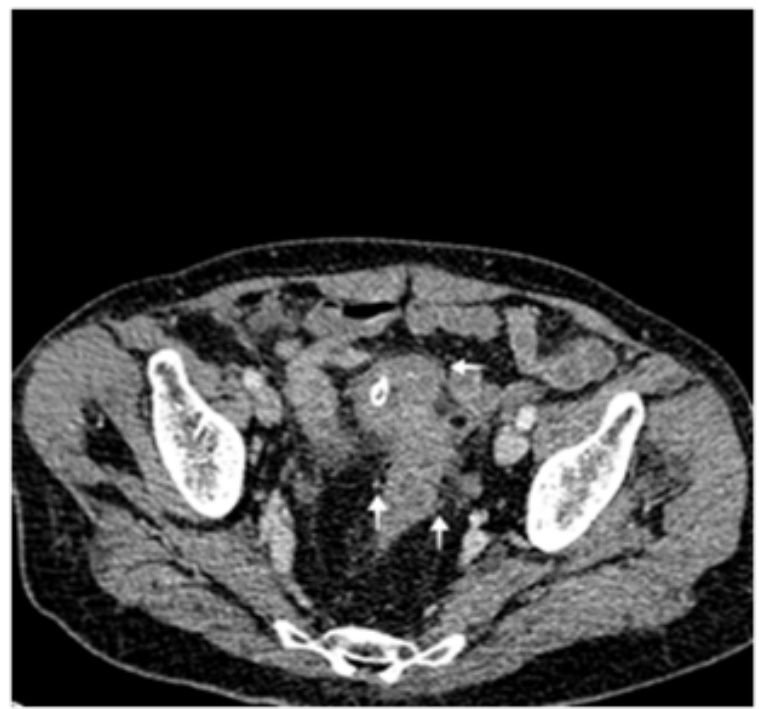

Figure 5 Transversal computed tomography scan showing majoration of bladder wall thickening nearby its contact with sigmoid colon. (Arrow $\longleftarrow$ )

The diagnosis of entero-vesical fistula is usually made by a bundle of clinical and paraclinical arguments. Clinical manifestation is usually non-specific, often leading to delay in diagnosis several months after symptoms begin. Pneumaturia and fecaluria, although pathognomonic of entero-vesical fistula, are present in only $50 \%$ of cases. ${ }^{1}$ Other much less specific signs such as suprapubic pain, irritative voiding symptoms, tenesmus, change of bowel habit, recurrent or polymicrobial urinary tract infection, malodorous urine, debris in the urine, or even epididymitis was related to entero-vesical fistula, thus making its diagnosis challenging. ${ }^{5-7}$

Radiological or endoscopic studies may sometimes be insufficient for the detection of an entero-vesical fistula with low reported average 
detection rate: cystoscopy $42.4 \%$; cystography, $41.3 \%$; barium enema, $35.3 \%$; colonoscopy $6.4 \%$; and abdominal computed tomography, $30.8 \%{ }^{6}$ In contrast to conventional invasive and expensive diagnosis methods that fail to diagnose entero-vesical fistula in up to $50 \%$ of cases, a simple and inexpensive diagnostic method that involves the oral intake of poppy seed can achieve $100 \%$ detection rate. The presence of indigested seeds in the urine is a positive confirmatory test for entero-vesical fistula. However, limits of this method are the absence of characterization of the fistula. ${ }^{6,7}$

Computed tomography is a satisfactory and reliable diagnosis study representing the modality of choice for diagnosis of enterovesical fistula, providing essential information about the adjacent anatomical structures and the underlying pathology causing the fistula thus guiding the therapeutic strategy. ${ }^{2,7}$ Endoscopic procedures (cystoscopy and colonoscopy) are very helpful in entero-vesical fistula diagnosis and determination of the underlying pathology by providing the possibility obtain a tissue biopsy to rule out malignancy and should be part of entero-vesical fistula workup. ${ }^{1,2,7}$

Usually, Because of high pressure in the intestine, the fistula works from the intestine to the bladder except when there is an obstacle on the lower urinary tract, as was the case of our patient resulting in opacification of the intestine during cystography thus permitting diagnosis of entro-vesical fistula.

Therapeutic management of entro-vesical fistula is largely dependent on the underlying pathology as well as the general condition of the patient. In clinical practice case by case strategy should be the rule. Surgical management became the preferred line of treatment with possibility of conservative approach in selected cases. $^{7}$ Means of Conservative approach include a trial of medical therapy as bowel rest, total parenteral nutrition, antibiotics, steroids, immunomodulatory drugs, and urethral catheter drainage. ${ }^{2}$ Single stage surgery is actually the treatment of choice. This entails resection of the diseased bowel segment, a partial cystectomy, primary bowel anastomosis and bladder wall repair followed by use of a Foley catheter for 1 week. . $3,3,7^{2}$

The old Staged procedure that entails initial colostomy, surgical repair and restoration of gastrointestinal continuity may be needed in cases with larges abscess or underlying malignancy. ${ }^{2,3,7}$

As our case expose and as reported in previous series outcome of entero-vesical fistulae management is, in the majority of cases, excellent. ${ }^{1,2,6,7}$

Minimally invasive techniques are possible in surgical management of entero-vesical fistulae. Marney el al., ${ }^{8}$ reported a large serie of 15 patients of diverticular colovesical fistulas treated with a totally laparoscopic approach including intracorporeal bowel anastomosis and bladder repair in wich they exposed laparoscopic management of diverticular colovesical fistulas as feasible and safe with acceptable operating times, morbidity, conversion rates, and excellent postoperative recovery if performed by skilled surgeons. Post-operative recurrence of entero-vesical fistulas is unusual in patients with benign and nonradiation- induced entero-vesical fistulae. Its occurrence especially if surgery was well performed should bring in mind the possibility of underlying malignancy.

\section{Conclusion}

Clinicians dealing with recurrent UTI therefore need to keep in mind this uncommon disease entity. Diagnostic tools include imaging studies and endoscopic procedures. Although conservative management can be attempted in selected patients, in most cases, the treatment is mainly based on surgical interventions recently moving toward a single stage surgery with literature data exposing good outcomes.

\section{Acknowledgements}

None.

\section{Conflict of interest}

Author declares there is no conflict of interest in publishing the article.

\section{References}

1. Lahyani M, Jabbour Y, Karmouni $T$, et al. Vesico-sigmoid fistula complicating intestinal hydatid disease: report of a rare case. Pan Afr Med J. 2014;19:117

2. Golabek T, Szymanska A, Szopinski T, et al. Enterovesical Fistulae: Aetiology, Imaging, and Management. Gastroenterology Research and Practice. 2013;617967:8.

3. Vincent P, Bhalla V, Gill M, et al. A Case of Entero-vesical Fistula. Med J Armed Forces India. 2000;56(1):61-63.

4. Bedoui R, Maghrebi H, Dougaz W, et al. Colonovesical fistula complicating colonic diverticulitis. Tunis Med. 2012;90(2):184-185.

5. Arneill M, Hennessey DB, McKay D. Colovesical fistula presenting with epididymitis. BMJ Case Rep. 2013; bcr2013009291.

6. Yang HY, Sun WY, Lee TG, et al. A Case of Colovesical Fistula Induced by Sigmoid Diverticulitis. J Korean Soc Coloproctol. 2011;27(2):94-98.

7. El Haddad HM, Kassem MI, Sabry AA, et al. Surgical protocol and outcome for sigmoidovesical fistula secondary to diverticular disease of the left colon: A retrospective cohort study. Int J Surg. 2018;56:115-123.

8. Marney LA, Ho YH. Laparoscopic Management of Diverticular Colovesical Fistula: Experience in 15 Cases and Review of the Literature. Int Surg. 2013;98(2):101-109. 